\title{
Evolución de la glomerulonefritis aguda en 29 casos comprobados con biopsia renal. Seguimiento a los 8 años
}

\author{
Dr. Patricio Donoso L.' ; Dr. Eduardo González C. ${ }^{1}$; Dra. M. Elena Donoso I. ${ }^{1}$; \\ Dr. Salvador Vial $\mathrm{U}^{2}$; E.U. Carmen Cubillos $\mathrm{M}^{3}$

\section{Long term clinical follow up in children after acute glomerular nephritis}

Twenty nine $(64,4 \%$ of 45 children with documented acute glamenlar nephritis (AGN) whoose clinical, hystological inmunological and follow up studies up to 590 (mean) days were previouly reported, came back to a recall evaluation 8.07 years (xange $75 / 12$ to $89 / 12$ years) after their first admission at a mean age of 15.6 years (range 10 to $213 / 12$ years). No one have had late symptoms nor sign attributable to the initial disease. Normal arterjal pressure was recorded in all patienţs and only 3 had slight proteinuria in their long term follow up urine sample (range 0.26 to $1.04 \mathrm{~g} / 1$ but not in the next late samples. These results include 2 of 4 initial patients with abnomal finding in renal biopsies performed at the second year after the begining of symptoms, whoose actual evaluation was now normal. This study reinforces the good prognosis generaly assigned to AGN in children.

(Key words: acute glomenu lar nephritis, long term prognosis).

En una comunicación anterior entregamos los resultados de un estudio clínico, histopatológico e inmunológico de 45 ninfos que sufrieron glomerulonefritis aguda (GNA) comprobada con biopsia renal al comienzo de la enfermedad y a $\operatorname{los} 194$ y 590 días promedio de evoluciớn ${ }^{1}$.

En este trabajo se analizan los mismos pacientes después de transcurridos, en promedio, 8,07 años (rango $7 \mathrm{a} 5 \mathrm{~m}$ a $8 \mathrm{a} 9 \mathrm{~m}$ ), desde su episodio agudo.

\section{MATERIAL Y METODO}

Se citaron al hospital Exequiel González Cortés los 45 enfermos de GNA incluidos en la primera comuricación, lográndose ubicar y examinar a $29(64,4 \%$ entre el 25 de mayo y el 3 de junio de 1987.

El control consistió en una anamnesis cuidadosa respecto al estado de saluc, actividad física y escotar; alteraciones perceptibles en la orina; existencia de ede-

1. Departamento de Pediatria. División Ciencias Móaicas Sur, Facultad de Medicina, Universidad de Chile. Hospital Exequiel González Cortés.

2. Departamento de Nefrourología. Untuersidad Católica de Chile. mas y causas de consultas médicas realizadas después del alta de su episodio agudo. Se practicó examen físico que incluyó peso, talla, evaluación del estado de nutrición: medición de la presión arterial en posición de pie después de un minuto y en decúbito dorsal después de tres minutos. Además fueron citados para una segunda oportunidad con el fin de medir creatinina plasmátics, depuración de creatining y examinar la orina, cultivándok, observando su sedimento y contando los hematies; también se determinó proteinurir mędiante las técnicas señaladas en la primera comunicación ${ }^{1}$.

Aquellos pacientes que mostraron alguna alteración, quedaron en observación o se trataron hasta su normalización.

\section{RESULTADOS}

De los 29 niños controlados, 12 eran hombres y 17 mujeres; la edad promedio fue de 15,6 años (rango $10 \mathrm{a}-21 \mathrm{a} 3 \mathrm{~m}$ ).

En la tabla se presentan los antecedentes entregados por la anamnesis en cuanto a las enfermedades presentadas desde la nefritis aguda hasta este control.

El peso para la talla actual se comparó con las tablas $\mathrm{OMS}^{2}$ en los niños que midieron $145 \mathrm{~cm}$ o menos y con tabla ICNND $1963^{3}$ en los que midieron sobre $145 \mathrm{~cm}$. Tres hombres y 10 mujeres tuvieron peso adecuado o superior al 
Tabla

Antecedentes de la anamnesis

\begin{tabular}{lr}
\hline & $\begin{array}{r}\text { No } \\
\text { casos }\end{array}$ \\
\hline Normales sin antecedentes & 9 \\
Amigdaltus crónica: asma & 3 \\
Síntomas de infección urinaria baja y/o & 5 \\
vulvovaginitis & 3 \\
"Dolor a'los riñones" y "lumbago" & 1 \\
Enuresis noctuma (a los 12 años) & 1 \\
Traumatismo renal & 1 \\
Edema facial ocasional hasta un año & \\
después de la nefritis & 6 \\
Otros (úlcera péptica, psoriasis, neumonia & \\
2 yaricelas, varicocele) &
\end{tabular}

percentil 50 y en 9 hombres y 7 mujeres éste fue menor que el aceptado para la altura.

La presión arterial, medida estando el paciente de pie y en decúbito, resultó en todo normal, bajo el precentil 95 de los valores del "Second Task Force"s4.

La creatinina plasmática promedio fue de 0,49 $\mathrm{mg} / 100 \mathrm{ml}$ (rango 0,21 a 0,67 ) y la depuración de creatinina fue en promedio de 153,6 $\mathrm{ml} / 1.73 \mathrm{~m}^{2}$ (rango 54 a 395).

El recuento promedio de hematies por campo microscópico fue normal (0 a 3 elementos) con una sola excepción que correspondió a un enfermo que presentaba infección urinaria, en el cual el recuento fue de 6 eritrocitos por campo.

Las proteinurias fueron negativas en 26 casos. En dos pacientes eran del orden de $0.26 \mathrm{~g} / \mathrm{l} \mathrm{y}$ en otro $1,04 \mathrm{~g} / \mathrm{l}$; los tres se controlaron posteriomente, resultando esta vez tămbién ne gativas.

Nos pareció particularmente interesante revisar los cuatro niños que en las biopsias alejadas del comienzo de la GNA presentaron signos histológicos de actividad (tres casos) o de cronicidad de las lesiones (un caso). Se pudo controlar a dos de ellos, ambos estaban asintomáticos, normotensos y uno, que habia tenido $8 \%$ de medialunas en la primera biopsia y numerosos glomérulos fibrosos en la biopsia al afio medio que nos hicieron suponer un mal pronósticos, tenía proteinuria de $0,26 \mathrm{~g} / 1$ y creatinina plasmática de $0,63 \mathrm{~g} / \mathrm{l}$ con depuración de creatinina de $169 \mathrm{~m} / 1,73 \mathrm{~m}^{2}, \sin$ hematuria: la proteinuria no apareció en controles posteriores. El segundo considerado histológicamente como lesiones activas, no tenia en este control proteinuria, habían 2 a 3 hematies por campo en su sedimento de orina y no aceptó hacerse examen de sangre. Un tercer caso, el considerado como crónico activo al salir del hospital, no acusó síntomas y está normotenso pero no concurrió al laboratorio para los exámenes de orina y de sangre.

\section{COMENTARIO}

Como sucede habitualmente en nuestro medio, hubo dificultades para ubicar los pacientes después de un tiempo, entre otras razones por cambios de domicibio. En este estudio pudo controlarse a $64,4 \%$, es decir, un tercio no fue ubicado. Esta es una limitante, sin duda, en el seguimiento de esta serie.

Los síntomas que presentaron parecen muy dificiles de relacionar con el episodio de nefritis; tal vez el único hecho importante es que un caso había tenido edema facial ocasional hasta un año después de la nefritis según observación proporcionada por la madre.

La evaluación del estado nutritivo debimos hacerla utilizando dos tablas diferentes debido a la edad de estos casos, intermedia entre niños y adultos. Es difícil evaluar nivel de desarnollo en este período de la vida, por los cambios hormorales que le caracterizan.

Debe destacarse que los 29 pacientes controlados tenían presiones arterjales normales y sólo tres tuvieron proteinuria en el examen de control inicial que no persistió en los controles posteriores por lo que es difícil interpretar su real significación.

Este control a los 8 años, aunque con las limitaciones impuestas por la pérdida de un tercio de ellos, permite apoyar la posición generalmente más aceptada, del buen pronóstico alejado de la glomerulonefritis aguda post estrepto. cócica que se presenta en la infancia.

\section{RESUMEN}

Se controlan 29 pacientes, de edaci promedio 15,6 años (rango 10 a 21 a $3 \mathrm{~m}$ ) que estuvieron hospitalizados por glomerulonefritis aguda post estreptocócica 8 años antes y que fucron motivo entonces de un estudio clínico, histopatológico e inmunológico ${ }^{1}$.

Desde la nefritis hasta el control actual, nueve estuvieron absolutamente sanos; los 20 restantes relatan algunos síntomas difíciles de relacionar con el episodio agudo. Trece presentan un peso 
adecuado a la talla y el resto está bajo el percentil 50 con respecto a los valores de las tablas OMS y de los valores promedios ICNND 1963.

Todo están normotensos en este control y presentan creatinina plasmática y depuración de creatinina normales. Sólo tres casos tienen proteinuria; de $0,26 \mathrm{~g}$ en dos y de $1,04 \mathrm{~g} / \mathrm{I} 100 \mathrm{en}$ otro; pero en exámenes posteriores las proteinurias fueron negativas.

Se pudo examinar a dos pacientes de los cuatro que en la biopsia alejada de la nefritis, aparecjan como activos o crónico; en ellos el examen actual es normal.
Se concluye que este seguirniento de GNA apoya la posición del buen pronóstico de esta enfermedad cuando se presenta en la infancia.

\section{REFERENCIA}

1. Vial S., Donoso P., Rosemberg $H$. et al: La glomerulonefritis aguda en el nix̌o. Rev Chil Pediatr 1987; 58: $11-28$

2. $O M . S$. : Medición del cambio del estado nutricional. 1983.

3. LC.NN.D.1963. Peso aceptable para aduitos.

4. Report of the Second Task Force on blood pressure control in children 1987. Pediatrics 1987; 79: 1-25. 